\title{
A survey of wireless ATM MAC protocols
}

\author{
Daniel Søbirk, Johan M Karlsson \\ Department of Communication Systems, Lund University \\ P.O. Box 118, SE-221 00 LUND, Sweden \\ Ph: +46-4622200 00, Fax: +46-46145823 \\ \{daniels, johan\}@tts.lth.se
}

\begin{abstract}
Recently there has been a considerable research interest in the next generation mobile networks. The new networks promises to be able to handle multi-service (voice, data, video) traffic. For fixed networks ATM is considered as the enabling technology for this traffic, so to ease the transition, as well as aid simple interworking, it is natural to use ATM for the mobile network as well. To support mobile applications ATM has to be extended with mobility support, a logical link layer, and a medium access control layer. This paper concentrates on medium access control issues, such as handling the different ATM services (CBR, VBR, ABR and UBR) with their different performance requirements.

This paper presents a survey of the current literature on MAC protocols and reports on the latest standardisation attempts. The authors present some known, and suggest several new, requirements on ATM MAC protocols. Furthermore a set of attributes suitable for describing generic MAC protocols are developed. Using these requirements and attributes, the most promising protocol proposals are compared and their respective technical merits are evaluated. Finally, a model for simulating MAC protocols on the broadcast radio medium is proposed and some preliminary results are reported and commented on.
\end{abstract}




\section{INTRODUCTION}

The public network operators (PNOs) are rapidly changing from being single service (telephony) providers to become multi service companies. Among the new, and coming, services offered are mobility (terminal and personal), freephone and other IN services, different computer communications services including Internet access, and various multi-media services, including such diverse applications as video conferencing and remote gaming. One of the enabling technologies for this transition is the Broadband ISDN (B-ISDN), standardized by ITU-T (1988). ITU has chosen Asynchronous Transfer Mode, ATM (ITU-T 1993), as the transfer protocol to be used by B-ISDN. The central characteristic of ATM are the use of small fixed size packets, called cells. The small packet sizes enables ATM to be used for voice as well as video and data transmissions, while the use of fixed size packets simplifies the implementation of high speed switches. Due to the promising performance potential of ATM, the computer communication industry has adopted the technology and ATM based LANs are becoming popular. Also, ATM may be used in the Internet backbone.

Since ATM was initially developed for B-ISDN several assumptions about the transmission medium were, properly, made. It was believed that ATM would be used on fiberoptic point to point links, providing high bandwidth and very low error rates. Consequently, there are no provisions for multiple access within ATM, and support for error control/detection is minimal and limited to the ATM header (Stallings 1994). However, the recent trend towards terminal mobility has spawned interest in wireless ATM implementations. The ambition is to provide a high bandwidth communication service, similar to B-ISDN, for mobile users. However, it is not expected that initial implementations are able to support full featured mobility (as in e.g. GSM), but rather provide isolated access islands. Even so, the first versions of the technology should allow a seamless evolution to a full-featured mobile broadband network, integrated with (or a part of) B-ISDN. To enable this evolution there is a need for standardising the wireless access, as well as mobility management in ATM.

This paper formulates and presents requirements on wireless ATM LAN Medium Access Control (MAC). A list of attributes of MAC protocols is also developed. The authors compare five of the more promising protocol proposals and evaluate their respective technical merits in terms of the previously defined requirements and attributes. Furthermore a simulation model suitable for capturing all the physical aspects of a wireless ATM LAN is briefly outlined and some preliminary results are reported. The paper also contains an extensive reference list.

\section{ATM AND MOBILITY}

During the past decade a tremendous amount of research has been performed on ATM. ATM is now accepted as the transmission mode to be used for B-ISDN, and is also rapidly gaining popularity in the computer communications world. As of now, 
PNOs are starting to deploy ATM in their fixed networks, and computer communications vendors are selling ATM equipment designed to replace existing LAN installations.

Hitherto unrelated to this vast interest in ATM, the telecommunications world has seen another strong trend during the last few years, namely mobility. Cellular systems such as GSM have enjoyed widespread consumer acceptance and are now widely deployed. Also wireless access systems, e.g. DECT, are beginning to gain popularity. The present day systems are characterized by providing low bandwidth services, typically telephony, in an environment with rather large cells. The large cell size is important, since it entails that handovers are infrequent.

The success for the mobile networks has, of course, spawned great plans for the next generation systems. It is expected that the next generation systems will provide the infrastructure for mobile multimedia applications, requiring significantly higher bandwidths than what is available today. Since the fixed counter part, B-ISDN, will use ATM, it is natural to assume that ATM will also be used to some extent in the next generation mobile networks. Initially ATM may only be used for the communication between the network and the base station, but eventually ATM will likely be used for the base station to mobile terminal communication as well.

\subsection{Wireless ATM}

ATM was developed to be used with virtually unlimited bandwidth, low bit error rate, point to point links. The radio medium, used in mobile networks, is inherently of a limited bandwidth, high bit error rate, broadcast nature. However, by using small cells and radio access in the gigahertz band, the limited bandwidth can be overcome, providing up to $30 \mathrm{Mbps}$ per cell. Advanced channel coding and ARQ schemes in a Logical Link Control Protocol can handle the high error rates, and a Medium Access Control Protocol can supervise use of the broadcast medium. Thus, ATM to the mobile terminal is a feasible endeavour.

A proposed protocol stack for wireless ATM (Raychaudhuri 1996) is outlined in

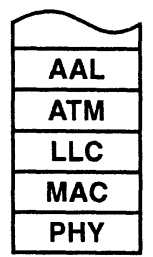

Figure 1 Possible protocol stack for wireless ATM. The AAL and ATM layers are similar to wireline ATM (mobility support has to be added). The MAC (Medium Access Control), LLC (Logical Link Control) and PHYsical layers are specific for the wireless implementation.

Figure 1. The usual AAL and ATM layers have to be extended with mobility support, and a Medium Access Control and a Logical Link Control (LLC) layer is added to handle the radio medium. This paper will concentrate on the MAC proto- 
col layer, hence the mobility support in the AAL/ATM layers as well as the LLC and physical aspects of wireless ATM is considered to be out of scope. Further overview information on wireless ATM systems can be found in (Acampora 1996, Ayanoglu 1996, Raychaudhuri 1996).

\subsection{Standards}

A lot of work on wireless issues is performed in different standardisation bodies and companies. Within ETSI the RES10 group has defined specifications for a wireless LAN, HIPERLAN type 1 (Wilkinson 1995), which was approved as a standard in 1996. However, HIPERLAN type 1 is not suitable for time bounded services, and therefore RES10 has started to work on three new standards for wireless ATM. These standards for wireless ATM will cover the lower layers of the OSI-model. Currently the work is in an early phase and the group has written a technical report covering requirements and architectures for these new standards (ETSI 1997). There are also a number of ACTS projects devoted to the subject.

In June 1996 the ATM Forum approved a study group devoted to wireless ATM, WATM. WATM will concentrate on network aspects of wireless ATM such as handover and location management.

In the U.S. the FCC has permitted use of parts of the $5 \mathrm{GHz}$ band for the UNII (Unlicensed National Information Infrastructure). The transmitted power and gain is regulated, but there are no requirements on spectrum etiquette or channelization, since the FCC considers these immature areas.

There are already wireless LANs on the market, such as Altair from Motorola and WaveLAN from AT\&T, but the data rates are too low for ATM and they have no support for time bounded services. IEEE 802.11 is working on a standard for wireless LAN, but also here the data rates are too low for ATM.

Naturally there are companies which have prototypes for wireless ATM networks. Olivetti Research has a prototype in Cambridge (Porter 1994, Olivetti 1995, Porter 1996) and NEC has one in the U.S. It is important that standards and products for wireless ATM can reach a large market and therefore wireless ATM network should be used in different environments and scenarios. One way of achieving this goal is to design a system that can be used in private, public and semi-public networks.

\subsection{Access algorithms}

A number of papers on MAC algorithms have been proposed. Most of the algorithms describe a type of TDMA-system in which the frames are divided in two parts: one contention part in which the mobile stations contend for access and one part in which the information is transmitted (Chou 1995, Karol 1995a, Karol 1995b, LaMaire 1994, Li 1995, Liu 1996, Narasimhan 1995, Petras 1995, Porter 1996, Tasaka 1995, Wen 1995, Wu 1995). In some algorithms it is the information packets that directly contends for the medium access (Babich 1994, Cleary 1995, Enomoto 1995, Modiano 1995, Papantoni 1994, Porter 1994, Roberts 1975, Sugihara 1995, 
Zhuang 1995). Some protocols can be considered as centralized polling protocols (Apostolas 1995, Karol 1995a, Karol 1995b, Liu 1996, Petra 1995, Smulders 1994). Only a few algorithms claim that they handle some kind of integrated traffic (Apostolas 1995, Babich 1994, Narasimhan 1995, Petras 1995, Smulders 1994, Tasaka 1995, Wu 1995). Of these algorithms only three have considered the different traffic types from a general point of view (Apostolas 1995, Petras 1995, Smulders 1994).

\section{MEDIUM ACCESS CONTROL IN WIRELESS ATM}

Medium access control for wired LANs is a well known area, for instance the IEEE has defined several MAC standards, of which CSMA/CD (Carrier Sense, Multiple Access with Collision Detect) and Token Ring are perhaps the most used. For wireless LANs these standards are not obviously useable, e.g. the use of carrier sense is probably not effective in a radio environment. Thus, there is a need to define new protocols, specifically designed for the wireless ATM environment with small packet sizes and high bandwidth requirements.

Then, what is the duties of the medium access control? Stallings (1994) defines the medium access control as: 'For broadcast networks, the method of determining which device has access to the transmission medium at any time.' This can be achieved with a variety of methods, including centralised, distributed and combined methods. This section will present requirements on the medium access control, specifically requirements for a MAC that is used in a wireless multimedia LAN. Furthermore, several attributes, suitable for classification of MAC protocols, are introduced. Finally, the differences between requirements and attributes are investigated.

\subsection{Requirements}

The function of the medium access control is to direct the use of the common, broadcast, medium among several stations. As illustrated in Figure 1 the MAC layer supports use of a shared medium for higher layers. Furthermore, cell sequencing, error detection and correction, retransmission etc. is handled by the LLC layer, directly above the MAC layer. Thus the MAC layer need only to be concerned with distributing access to the medium.

It is desirable if this control fulfils the following natural requirements. Access to the medium should be granted to the stations in a "fair" manner, different traffic types (e.g. real-time video, batch data transmission) should be handled keeping their respective Quality of Service (QoS) demand, while the utilization of the medium should be high. These requirements apply to all MAC protocols, both for wireless and wireline environments (as well as other environments, if they can be imagined).

For wireless LANs the IEEE has formed a study group (IEEE 802.11) that further investigated the requirements for this special application. They formulated 20 requirements (Chen 1994) on a general wireless MAC layer. We have evaluated these requirements for our specific application, an ATM based wireless LAN for 
public access, and tried to rate the requirements' order of importance. This rating is rather coarse, but e.g. throughput and delay are considered, in general, more important than items further down the list. Thus, the list of requirements is presented next, in a rough priority order, with the most important requirements listed first.

1. Throughput.

2. Delay.

3. Ability to serve data, voice and video.

4. Preservation of packet order.

5. Ability to support handoff/roaming between service areas.

6. Robustness in collocated networks.

7. Support priority traffic.

8. Ability to support nonreciprocal (e.g. downstream larger than upstream) traffic.

9. Battery power consumption.

10. Fairness of access.

11. Maximum number of nodes.

12. Ability to work in a wide range of systems.

13. Transparent to different physical layers.

14. Unauthorized network access impact on throughput.

15. Ability to support broadcast (multicast).

16. Establish peer-to-peer connectivity without a priori knowledge.

17. Critical delays limit large area coverage.

18. Insensitivity to capture effects.

19. Limit the complexity of the physical layer.

20. Ability to market and complexity.

\section{Comments}

Throughput is, as always, a very important parameter of the protocol. In the case of wireless ATM LANs both the utilization of the allocated spectrum bandwidth must be high (i.e. bps/Hz) as well as the absolute throughput of ATM cells (e.g. at least $10 \mathrm{Mbps}$, not counting MAC overhead bits). The delay performance is critical since it is expected that the high bandwidth service will be used for multimedia services, especially video services. The ATM concept is designed to support a variety of services so this versatility should also be available in the wireless implementation. Moreover, the ATM concept relies on cells being delivered in the correct order, this property should not be broken in the wireless layers.

The priority order indicated in the list should not be considered an absolute order, it is rather an indication - requirements at the top of the list are more important than requirements at the bottom.

\section{Additional requirements}

The IEEE requirements described above, while satisfactory for a wireless LAN replacing e.g. an installed Ethernet, do, for example, not address the situation where 
a public operator offers wireless broadband access to a LAN environment as well as access to fixed networks (like the Internet). Thus, due to these and other additional new scenarios, several additional requirements are formulated by the authors. The list of requirements does not try to reflect any order of importance.

- Robustness. Due to the error prone medium, the protocol should not rely on nodes knowing the entire system state. In that case a node which, perhaps due to a local burst error, missed a reservation transmission might assume that the medium is free and transmits itself, leading to an unnecessary collision. The robustness requirement also implies that a case of sudden station shut down or disappearance must not affect the protocol performance.

- Base station. Apostolas (1995) makes several compelling arguments on the benefits of using a base station, e.g. with a base station an interconnection to a fixed network (e.g. the Internet) is easily implemented. Furthermore, a base station design can result in a more robust MAC protocol (see above).

- Bandwidth partition. It should be possible to divide the bandwidth in different parts for different user groups. Then private companies can use their own networks to offer public access, but still have some bandwidth reserved for their own users.

- Adaptation to upper layers. The MAC layer shouldn't lay any unnecessary burden on the upper layers.

- Frame size compatible with ATM. For easy interworking, the implemented frame size in the MAC protocol should be either a multiple or fraction of the ATM cell size.

- Non equidistant nodes. The MAC protocol shouldn't assume that the nodes are (geographically) equally distributed in the cell.

- Insensitive to cell transfer speed. In order to ease migration to higher speeds the MAC protocol should not make any assumptions about cell transfer speed.

- Fair and efficient contention resolution algorithm. Contention resolution must at least be used in the registration phase, and it is desirable to solve conflicts in an efficient and fair way.

- Mass market appeal. The cost of implementation is very important to generate a mass market appeal. In the age of micro-cellular structures it is not only the cost of the portable interface that is important, the base stations should be cheap to manufacture as well.

\subsection{Attributes}

The MAC protocols that have been proposed in the literature vary from rigorously controlled polling protocols e.g. (Smulders 1994), to the free-for-all Aloha protocol (Abrahamson 1970). To be able to compare such vastly different protocols, it is useful to define attributes or features of a MAC protocol, as well as, the attributes' possible values. These attributes should not be limited to wireless MAC, but are rather applicable on all MAC protocols. Please note that the values in themselves are not good or bad, it is the particular usage situation (noisy radio environments, satellite 
communications, and coaxial cables all have very different operating conditions) that determines whether a certain attribute value is desirable or not. Thus the attribute values of a certain protocol should be compared with the requirements for the particular usage situation to determine whether the protocol is suitable for that particular situation, or not.

The attributes are listed with their names and possible values first. In certain cases the possible values may indicate a range rather than discrete values. A brief explanation of the different values is given for each attribute.

1. Requires infrastructure. Yes/no. Yes usually means that a base station is needed to support communication between nodes. No indicates that ad hoc networking is possible.

2. Intelligence. Central/distributed/both/none. Central intelligence means that one node (e.g. the base station) keeps track of the system state and conducts the transmissions accordingly. A distributed intelligence scheme assumes that the individual stations maintain the system state, while divided responsibilities gets the value both. Of course, a MAC protocol can be "dumb", i.e. transmit whenever a packet is ready (cf. Aloha). This is referred to as none.

3. Up/downlink channels. Different/same. Some MAC protocols assume that the downlink traffic is handled on a different channel and thus only handles traffic from the stations to the base station, while others handles both up- and downlink traffic on the same channel.

4. Time division. Discrete/continuous. Refers to whether the protocol divides the time into discrete slots or not (cf. slotted Aloha vs. pure Aloha).

5. Main access strategy. Collision free/contention based. Whether the protocol is collision free or not, in a broad general sense.

6. Access request. With request packet/with data packet/via polling. When requesting access to the medium certain protocols specify that this must be done during a special time frame, with special request packets. If access is granted the actual packet is transmitted later. Other protocols request access with the actual data packet, if no collision occurs the packet is considered successfully transmitted. If access is granted via polling then none of the previous values apply, and the via polling value is used.

7. Reservation strategy. This attribute is rather complex so it has been divided into several subclasses.

a. First packet in a burst. Contention/reservation/fixed. A contention based scheme means that a new burst must compete with other pending transmissions, according to a strategy as specified in attribute 6 . A reservation approach assumes that a new burst request is made by the protocol, and a base station acknowledges this request, typical for a polling based protocol. The fixed allocation is analogous to a circuit switched connection, a specific time slot is always available for the station.

b. Remaining cells in a burst. None/burst reservation. A protocol may chose not to make any special consideration to bursts in the allocation algorithm. In 
that case, every packet must contend according to the principle in $a$. The fixed strategy in $a$ is inherently of this kind, but others exist, most notably adaptive polling schemes as well as pure contention methods. A burst reservation scheme, on the other hand, assumes that once a packet is transmitted successfully that a time slot is reserved for the use of the transmitting station for some time period (e.g. until it is not used, or a specific maximum number of slots).

c. Bandwidth allocation flexibility. Contention/request/new connection. When a station demands more bandwidth it may simply attempt to transmit packets more often (contention), it may request permission to transmit more frequently (request), or it may have to request an entirely new connection. The last method is typical for circuit switched connections. Also refers to how a station which has lowered its bandwidth demands may return any allocated resources.

8. Contention resolution strategy. None/random binary backofflothers. If a collision occurs, all the involved stations must not try to retransmit at the same time, or a collision will occur again. The contention resolution strategy describes how the stations should behave in order to minimize the risk that a collision occurs at the retransmission attempts. Several algorithms are described in the literature (Bertsekas 1987), and the value of this attribute just names the used strategy, if any.

9. Multiplexing technique. CDMA/TDMA/FDMA/any. A properly designed MAC protocol should be largely independent of the physical layer multiplexing technique, nevertheless it is mentioned here since some protocols may take advantage of features in special multiplexing techniques (i.e. CDMA in itself can be considered a multiple access protocol).

10. Carrier sense. Yes/no. A protocol that tries to determine whether a transmission is in progress before it tries to transmit itself, utilises carrier sense.

11. Traffic integration. None/class based/seamless. Traffic integration refers to how the protocol handles data streams with different QoS requirements, e.g. voice vs. video vs. data streams. A protocol may chose not to distinguish the different streams, divide the streams into different classes based on QoS parameters (just one class is equal to none in this case), or a seamless integration with a scheduling algorithm selecting the packet to transmit.

12. Frame structure. Homogenous/heterogeneous. A MAC protocol organizes its transmissions in frames. A frame consists of several subslots which each could be used for data transmissions (reserved or contention), future reservations or registrations. If a frame consists entirely of data transmissions it is referred to as homogenous. The frame may also be divided into two or several phases (heterogeneous), e.g. a real-time traffic phase followed by a data traffic phase. In other protocols a frame may be divided into a registration phase, used for the registration of new stations, a control phase where stations may transmit control messages to a base station and a transmission phase where the actual data trans- 
missions take place. The access strategy used for the different phases is arbitrary. If the protocol uses a heterogeneous approach the names of the phases are mentioned.

\subsection{Requirements vs. Attributes}

It is important to note that requirements and attributes are rather unrelated concepts, requirements represent what a MAC protocol should do, attributes represent how a MAC protocol works. Thus a specific value of an attribute does not necessarily indicate that a specific requirement is fulfilled or not, rather the attributes are used to describe a MAC protocol. Then, all the attributes can be used to evaluate the protocol to see whether it fulfils certain requirements.

\section{SOME PROPOSED MAC PROTOCOLS}

This section presents some of the most interesting proposals for wireless ATM protocols. The protocols are explained in some detail, and at the end the protocols are compared in respect to the previously introduced requirements and attributes.

\subsection{Slotted Aloha}

Slotted Aloha (S-Aloha) (Roberts 1975) is based on the Aloha protocol (Abrahamson 1970). The Aloha protocol separates the downlink and uplink channel. On the uplink channel, the stations may transmit packets at any time. Successfully transmitted packets are broadcasted on the downlink by the base station, this serves as packet acknowledgement. Naturally the throughput for this scheme is low (Bertsekas 1987). Slotted Aloha improves on this by dividing the time into discrete slots, with the size equal to a frame. Transmissions are only allowed to start at the beginning of a slot. This effectively doubles the throughput (Bertsekas 1987) of the Aloha protocol. Although the S-Aloha protocol is not specifically developed for wireless ATM and has a rather low throughput it may be an attractive solution due to the simplicity of implementation.

\subsection{DQRUMA}

Distributed-Queueing Request Update Multiple Access (DQRUMA) (Karol 1995a, Karol 1995b, Liu 1996) is a MAC protocol designed for a wireless ATM LAN. The downlink is used for broadcasting packets and control information, and is considered to be separate from the uplink channel. The uplink channel is divided into Request Access (RA) phases and packet transmission phases, controlled by the base station via the downlink. Whenever a station wishes to transmit a packet, it uses a RA phase to transmit a request (possibly contending with other stations) to the base station. When the base station successfully receives the request it transmits an acknowledgement on the downlink and registers the station's request. The base sta- 
tion then distributes access to the uplink packet transmission phases, according to e.g. a round-robin fashion, by polling the stations via the downlink. Whenever a station is polled and transmits a packet it piggybacks an additional bit indicating whether it has any more packets to send. This serves as a contention free request. Furthermore, e.g. CBR connections may at call setup request that the station will be polled at periodic intervals, thus further avoiding contention in the RA phase.

In order to support seamless traffic integration the polling protocol should be changed from round-robin to a scheme that takes cell priority into account. This may, however, require some minor adjustments to the DQRUMA protocol, e.g. it would be beneficial for the polling protocol to know not only whether a station has a packet to send, but also to know how many packets there are in the buffer, and, perhaps, their residual life.

\subsection{Polling Protocol 1}

Apostolas (1995) presents a MAC protocol for an ATM based wireless LAN (from here on referred to as Apostolas). The protocol separates the downlink and uplink channel, where a base station broadcasts every transmitted packet on the downlink. The uplink is divided into four phases, transmission, transition to active, registration, and control. In the transmission phase ATM cells are transmitted in response to polling by the base station. The ATM header is extended with an extra status byte allowing stations to inform the base station of required bandwidth (more/less than currently allocated), a transition to inactive (the cell is the last in a burst), or a control request. In the transition to active phase all currently inactive connections are polled to allow further transmissions. The registration phase allows new terminals to register themselves to the network. This registration is, by necessity, done in a contention mode. A successful registration results in a assigned VPI number for the station and that a control channel is allocated for that specific terminal. The control phase polls the inactive terminals (and those who have issued a control request) for control messages (i.e. connection set up/removal, management information, etc.). The allocation algorithm used for determining which station (and connection) to poll at a given instant is not described, but it is expected that any polling algorithm designed for ATM-like traffic could be used, e.g. (Karlsson 1992).

\subsection{Polling Protocol 2}

Smulders (1994) introduces a MAC protocol for ATM in indoor radio networks (further referred to as Smulders). The protocol, similarly to Apostolas, separates the downlink and uplink, and divides the uplink into two phases, transmission and request. An uplink transmission occurs in response to a poll from the base station, and the transmitted MAC frame consists of an ATM cell and a number indicating the number of waiting cells at the mobile terminal. The base station uses this information to control the polling algorithm. During idle periods the base station starts a 
request phase. In this phase the base station polls the inactive terminals to transmit the number of waiting cells.

The base station maintains information on how many cells each station has waiting and when the station should be allowed to transmit the next time. This time is decided with the aid of negotiated (during call setup) peak cell rates, and the information on when the station was last allowed to transmit. Thus, the allocation (polling) algorithm enforces these negotiated peak cell rates by cell spacing. Consequently, the polling algorithm effectively works as a traffic shaper.

\subsection{DSA}

Another protocol named Dynamic Slot Assignment (DSA) is introduced by Petras (1995). This protocol separates the up- and downlink. The downlink is used both to broadcast packets and to transmit acknowledgements and polls (either one at a time as part of a broadcast cell, or an entire batch at a time). The terminals uses the uplink transmissions to inform the base station of the number of waiting cells and their smallest residual life. The base station uses this information as input to the polling algorithm. However, according to the inventors, this information is not enough when the number of cells (or their residual life) changes rapidly, so the protocol uses a special contention phase where stations in need are allowed to transmit cells according to S-Aloha. Also, inactive terminals use this contention phase to start the transition to active. The polling algorithm calculates priorities according to the number of waiting cells and their smallest residual life and assigns the next transmission slot to the station with the highest priority.

\subsection{GRAPO/R-GRAP}

Li (1995) presents GRAPO - Optimized Group Randomly Addressed Polling for wireless data networks, and Chou (1995) presents Group Randomly Addressed Polling with Reservation for wireless integrated service networks (R-GRAP). Both papers improves the GRAP (Group Randomly Addressed Polling, in turn an improvement of Randomly Addressed Polling) algorithm, in two different, although orthogonal, ways. We believe that both improvements can be incorporated into a "R-GRAPO" protocol, although the original authors (one of which is co-author on both papers) may not agree that it is necessary. Nevertheless, the two protocols are in principle so similar that they will be treated as one in this paper.

The Randomly Addressed Polling (RAP) protocol is a contention protocol which limits the number of simultaneous transmission attempts through a random selection. The protocol separates the up- and downlink channels, and is mainly concerned with the uplink. The base station transmits a ready message when it is ready to receive uplink messages. In response, each active node (in total $N$ active nodes) will choose and transmit a random number $(0,1, \ldots, p-1)$ in a way that allows the base station to receive all the transmitted numbers simultaneously. The base station will detect $N^{*}(\leq N)$ different numbers; for each number the base station will broad- 
cast this number, and the terminals which chose this number will transmit a packet. If several terminals chose the same number a collision will occur. The base station will provide feedback (ACK/NACK) and for the unsuccessful transmissions the procedure is repeated.

The Group Randomly Addressed Polling improves on RAP by introducing a superframe structure consisting of $p+1$ frames, named $\mathrm{G} 0, \mathrm{G} 1, \ldots, \mathrm{G} p$. Each frame is a separate RAP frame. A new node will join frame $\mathrm{G} p$, whereas a node which in the previous superframe was successfully polled with the random number $i$ (and has more packets to transmit) will join frame Gi in the current superframe. GRAPO further improves on this concept by dynamically change the number of groups to $Q$ $(\leq p)$, and requires new arrivals to join a random group. Successfully polled terminals stay in the same group, with the same random number. Stations that are unsuccessfully polled are treated as new arrivals. On the other hand, R-GRAP improves on GRAP by introducing a separate phase, called the reservation phase. When a terminal has periodic or long data to send it will transmit the first packet according to the GRAP protocol, with a special bit set, which indicates that more data follows. If the packet is successfully transmitted the terminal will not only receive an ACK from the base station, but also a reservation number. These reservation numbers are used after the last GRAP frame, when the reservation phase begins. In the reservation phase, the base station will poll all (or some, according to an allocation algorithm) reservation numbers and the stations can transmit their frames in a contention free way.

\subsection{Comparison}

In this section, the protocols presented above are compared. The protocols' fulfilment of a crucial subset of the requirements are evaluated (Table 1) and the protocols' attributes (Table 2) are included.

Table 1 Fulfilment of some requirements for six different MAC protocols

\begin{tabular}{lllllll}
\hline Requirement & S-ALOHA & DQRUMA & Apostolas & Smulders & DSA & GRAPO \\
\hline Throughput & low & high & unknown & high & high & high \\
Delay & high & moderate & moderate & unknown & moderate moderate \\
Data, voice, video & no & yes & yes & yes & yes & yes? \\
Packet order & yes & yes & yes & yes & yes & yes \\
Peer-to-peer & yes & yes & yes & yes & yes & yes \\
Priority traffic & no & no & yes & yes & yes & no \\
Non-reciprocal & yes & yes & yes & yes & yes & yes \\
Number of nodes & no limit & address bits & VPI address address bits & no limit & no limit \\
PHY reqs & yes & yes & yes & yes & yes & CDMA? \\
Multicast & BS & BS & BS & BS & BS & BS \\
\hline
\end{tabular}


Table 1 Fulfilment of some requirements for six different MAC protocols

\begin{tabular}{|c|c|c|c|c|c|c|}
\hline Requirement & $S-A L O H A$ & $D Q R U M A$ & Apostolas & Smulders & $D S A$ & GRAPO \\
\hline $\begin{array}{l}\text { Complexity of } \\
\text { PHY }\end{array}$ & yes & yes & yes & yes & yes & advanced \\
\hline Robustness & high & high & high & high & high & high \\
\hline Base station & yes & yes & yes & yes & yes & yes \\
\hline $\begin{array}{l}\text { Adapt to upper } \\
\text { layers }\end{array}$ & ok & ok & ok & ok & ok & ok \\
\hline ATM frame size & yes & yes & yes & yes & yes & yes \\
\hline $\begin{array}{l}\text { Non equidistant } \\
\text { nodes }\end{array}$ & ok & ok & ok & ok & ok & ok \\
\hline $\begin{array}{l}\text { Cell transfer } \\
\text { speed }\end{array}$ & yes & yes & yes & yes & yes & yes \\
\hline $\begin{array}{l}\text { Fair and efficient } \\
\text { contention resolu- } \\
\text { tion algorithm }\end{array}$ & no & $\mathrm{N} / \mathrm{S}$ & $\mathrm{N} / \mathrm{S}$ & $\mathrm{N} / \mathrm{S}$ & $\mathrm{N} / \mathrm{S}$ & yes \\
\hline $\begin{array}{l}\text { Mass market } \\
\text { appeal }\end{array}$ & yes & yes & yes & yes & yes & moderate \\
\hline $\begin{array}{l}\text { Bandwidth parti- } \\
\text { tion }\end{array}$ & no & yes & yes & yes & yes & no \\
\hline
\end{tabular}

Table 2 Attributes of six different MAC protocols

\begin{tabular}{|c|c|c|c|c|c|c|}
\hline Attribute & $S-A L O H A$ & $D Q R U M A$ & Apostolas & Smulders & $D S A$ & GRAPO \\
\hline Req infrastructure & yes & yes & yes & yes & yes & yes \\
\hline Intelligence & none & central & central & central & central & both \\
\hline Up/downlink & different & different & different & different & different & same \\
\hline Time division & discrete & discrete & discrete & discrete & discrete & discrete \\
\hline Access strategy & contention & coll. free & coll. free & coll. free & coll. free & contention \\
\hline Access request & data & req & polling & polling & polling & data \\
\hline \multicolumn{7}{|c|}{ Burst reservation strategy } \\
\hline First cell & contention & contention & reservation & reservation & contention & contention \\
\hline Remaining cells & contention & burst res & none & none & burst res & none \\
\hline BW alloc flex & contention & $? ? ?$ & request & request & request & contention \\
\hline Cont res strategy & $\mathrm{N} / \mathrm{S}$ & $\mathrm{N} / \mathrm{S}$ & $\mathrm{N} / \mathrm{S}$ & $\mathrm{N} / \mathrm{S}$ & $\mathrm{N} / \mathrm{S}$ & RAP \\
\hline Multiplexing & any & any & any & any & any & CDMA? \\
\hline Carrier sense & no & no & no & no & no & no \\
\hline Traffic integration & none & seamless & seamless & seamless & seamless & none \\
\hline Frame structure & $\begin{array}{l}\text { Homo- } \\
\text { genous }\end{array}$ & $\begin{array}{l}\text { Hetero- } \\
\text { geneous }\end{array}$ & $\begin{array}{l}\text { Hetero- } \\
\text { geneous }\end{array}$ & $\begin{array}{l}\text { Hetero- } \\
\text { geneous }\end{array}$ & $\begin{array}{l}\text { Hetero- } \\
\text { geneous }\end{array}$ & $\begin{array}{l}\text { Hetero- } \\
\text { geneous }\end{array}$ \\
\hline
\end{tabular}


Generally the polling protocols (DQRUMA, Apostolas, Smulders, DSA) are rather similar and can be expected to have similar performance characteristics, possibly more influenced by the choice of polling function than the chosen medium access strategy. Still, there are some interesting differences in the request/transition to active procedures that might influence the delay performance. The GRAPO/RGRAP protocol, on the other hand, introduces a radically different approach. There are questions as to whether the protocol will be able to serve time dependent data like voice or video, and if a class based (and only two classes to boot) traffic integration concept will be enough for the complex ATM traffic situation envisioned.

\section{PERFORMANCE EVALUATION}

One of the most important aspects of the medium access control is the performance, e.g. the maximum achievable throughput or the delay characteristics. While it is desirable to develop these results with analytical calculations, it is often not feasible. Complex (i.e. real-world multimedia) input traffic, and hard to model (analytically) protocols make analytical solutions cumbersome at best.

To further investigate the utilization and delay characteristics for the MAC protocols, the authors have developed a simulation model. The model accurately describes the error-prone medium with geographically distributed stations. Additionally, the input traffic to the model can be taken from measurements (Björkman 1995), thus ensuring that the results reflect real world performance. Due to the nature of simulation, the results only reflect the performance of the MAC under one (or a few) specific situations, it is not recommended to draw extensive conclusions from isolated simulation results. However, general trends can certainly be identified.

\subsection{The model}

The simulation model is developed to allow easy interchange of MAC protocols, accurately model the physical behaviour of the broadcast channel, including precise error modelling, and a choice of having arrivals according to an arbitrary random arrival process or from measurements. The model is comprised of four processes, User, User MAC, optionally the Base Station MAC, and the Medium processes. The model is further described in (Johansson 1997).

\subsection{Preliminary results}

This section presents some preliminary results from the simulated model. Presently only S-Aloha, DQRUMA, Smulders and GRAPO are simulated; some vital details in the descriptions of Apostolas and DSA are missing, thereby hindering implementation of these.

The capacity of the channel was set to $25 \mathrm{Mbps}$ and the users were (randomly) spread out within a circle with a diameter of $70 \mathrm{~m}$. Somewhat unrealistic, no errors 
were simulated. Each user is in one of two states, idle or sending a file with FTP. The mean time spent in the idle state was 3 seconds. The size of the transferred file was chosen from the exponential distribution with a mean of 400.000 cells. The exact traffic pattern was modelled according to the measurement in (Björkman 1995), i.e. the cells were generated in bursts. The number of users was varied from 1 to 30 (15 for S-Aloha), i.e. the offered load varied from 0.05 to $1.46(0.73)$.

Figure 2 plots the utilization (defined as the fraction of time the channel is used
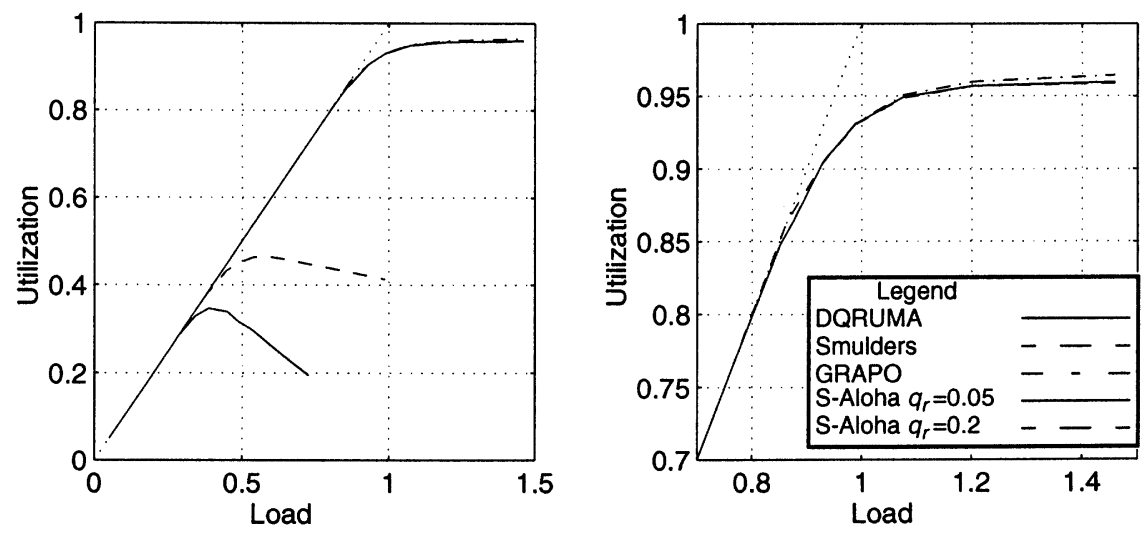

Figure 2 Utilization for FTP traffic. The dotted line indicates the ideal utilization. For S-Aloha, the probability that the protocol will try to retransmit in a timeslot is denoted $q_{r}$

for transporting pure ATM-cells, as opposed to e.g. polling data or MAC-frame overhead bits) as a function of the offered load for the four different MAC protocols when the number of users is kept constant. As expected, S-Aloha performs very poorly, adequately handling only loads less than 0.3 . The other protocols perform similarly, and manage to handle loads up to 0.85 . It is evident that the protocols do not suffer from performance degradation during overload conditions, rather the utilization remains constant.

The delay (defined as the time from the cell is generated by a user until it is delivered to the base station) is shown in Figure 3. As expected, the delay rises sharply at the point where the protocols become overloaded. From this point the delays continue to increase due to the increased number of users demanding access to the channel. Once again, S-Aloha performs significantly worse than the others, which, on the other hand, perform remarkably similar.

\subsection{Analysis}

Generally it can be seen that for the FTP traffic examined here, the protocols can be divided into two groups, S-Aloha and the rest. S-Aloha performs significantly worse than the others for all loads larger than 0.3 . The three other protocols, how- 


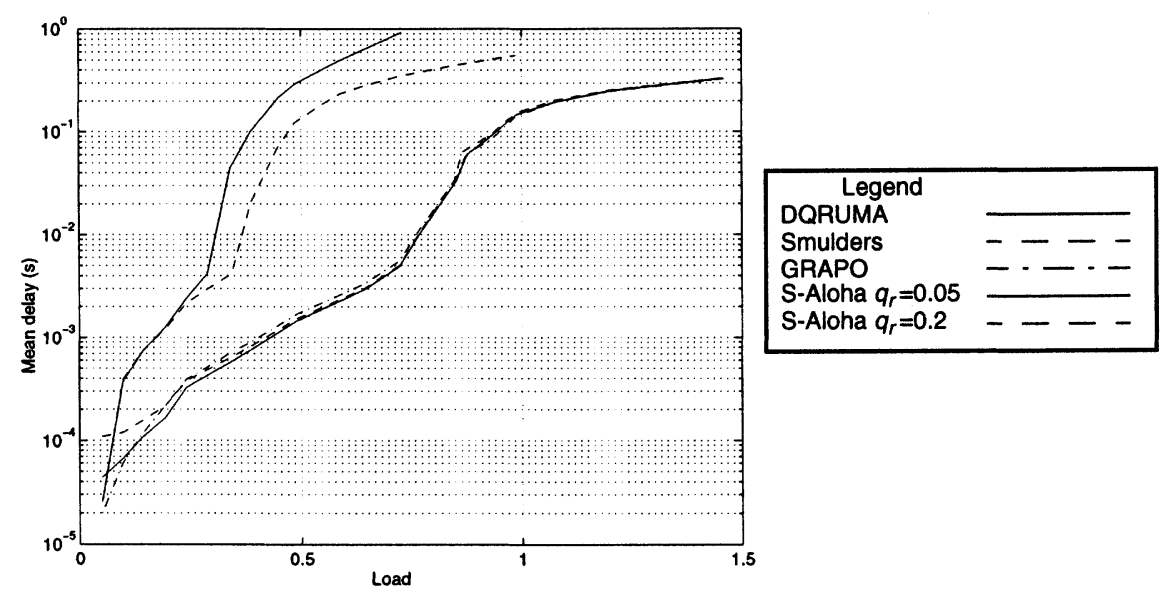

Figure 3 Delay for FTP traffic.

ever, exhibit very similar behaviour, with differences only noticeable at very high loads.

It is stressed that, as stated previously, all the results in this report are for FTP traffic only. For other traffic arrival distributions, specifically mixed traffic scenarios, further studies are required. Such studies are, at the moment, being performed by the authors.

\section{CONCLUSIONS}

The rapidly growing consumer demand for all aspects of wireless networking has generated a great interest for the next generation systems. It is expected that the next generation mobile systems will provide a mobile counterpart to B-ISDN, a service integrated broadband network. Since it is likely that this mobile network will eventually be integrated with B-ISDN it is natural to use ATM as the transport technology for the new mobile network too. To accomplish this ATM must be extended with mobility features, a data link layer, and a medium access control layer. Naturally, the performance of these layers are crucial for the success of wireless ATM. This paper concentrates on the medium access control layer and formulates requirements and attributes, compares several proposed MAC proposals and evaluates their performance.

The IEEE study group 802.11 has studied MAC protocols for wireless LANs, and in this process they have developed a set of requirements. These requirements are briefly presented in this report, and also prioritized with a wireless ATM implementation in mind. Additionally, the authors have formulated a number of new requirements with emphasis on the special needs when using ATM technology. Furthermore, a set of attributes (with related values), chosen to accurately model a 
MAC protocol, is proposed. It is expected that these attributes may ease the comparison of MAC protocols.

The, in the authors' view, most promising MAC protocols proposed in the literature are described in further details. They are also compared in respect of the requirements and attributes described in the paper, using S-Aloha as a reference protocol. Finally, a simulation model, designed to capture the specific properties of a wireless environment, for a MAC protocol is defined. The utilization and delay for a number of the described protocols when handling FTP traffic are reported on. The results show that the recent protocol proposals significantly outperform the classical S-Aloha protocol. Further work is needed to evaluate how the proposed protocols handle e.g. real-time traffic and mixtures of different traffic sources.

\section{ACKNOWLEDGEMENTS}

The authors would like to express their gratitude to Lars Falk and Christer Lind of Telia Research, Sweden, for their contribution to section 2 and general cooperation with this work. Furthermore, the implementation of the simulation model was done by Christian Johansson, Henrik Nilsson, and Rickard Rothpfeffer, for which the authors are grateful.

\section{REFERENCES}

Abrahamson, N. (1970) The ALOHA system-Another Alternative for Computer Communication, in Proc. Fall Joint Computer Conference.

Acampora, A. (1996) Wireless ATM: A Perspective on Issues and Prospects, IEEE Personal Comm., Vol. 3, No 4, 8-17

Apostolas, C., Tafazolli, R., and Evans, B. (1995) Wireless ATM LAN, in Proc. of the 6th IEEE International Symp. on Personal, Indoor and Mobile Radio Comm., 773-777.

Ayanoglu, E., Eng, K. Y. and Karol, M. J. (1996) Wireless ATM: Limits, Challenges, and Proposals, in IEEE Personal Comm., Vol. 3, No 4, 18-33

Babich, F. (1994) Analysis of a Contention Resolution Multiple-Access Algorithm for Handling Traffic on Wireless Networks, in Int. J. of Wireless Information Networks, Vol. 1, No. 4, 271-287.

Bertsekas, D. and Gallagher, R. (1987) Data Networks, Prentice-Hall Inc.

Björkman, N., Hansson, U., Latour-Henner, A., Pers, O: and Miah, A. (1995) Practical ATM Resource Dimensioning Based on Real-time Traffic Measurements and Analysis, in Proc. of Globecom'95, Vol. 1, 399-403.

Chen, K-C. (1994) Medium Access Control of Wireless LANs for Mobile Computing, in IEEE Network, Vol. 8, No. 5, 50-63. 
Chou, H., Lee, C. and Chen, K. (1995) Group Randomly Addressed Polling with reservation for Wireless Integrated Service Network, in Proc. of the 6th IEEE International Symp. on Personal, Indoor and Mobile Radio Comm. , 618-622.

Cleary, A. and Paterakis, M. (1995) An Investigation of Reservation Random Access Algorithms for Voice-Data Integration in Microcellular Wireless Environments, in Int. J. of Wireless Information Networks, Vol. 2, No. 1, 1-16.

Enomoto, K. , Shiokawa, S. and Sasase, I. (1995) Performance Analysis of Idle Signal Multiple Access with Multiple Idle Signals, in Proc. of the 6th IEEE International Symp. on Personal, Indoor and Mobile Radio Comm., 643-647.

ETSI TC-RES/RES10 Draft Technical Report (1997), HIgh PErformance Radio Local Area Networks (HIPERLANs) Requirements and Architectures for Wireless ATM Access and Interconnection.

ITU-T Recommendation I.121 (1988) Broadband Aspects of ISDN.

ITU-T Recommendation I.150 (1993) B-ISDN Asynchronous Transfer Mode Functional Characteristics.

Johansson, C., Nilsson, H. and Rothpfeffer, R. (1997) Comparison of MAC Protocols for Wireless ATM (in Swedish), M. Sc. Thesis, Dept. of Communication Systems, Lund University.

Karlsson, J.M., Perros, H.G. and Viniotis, I. (1992) Adaptive Polling Schemes for an ATM Bus with Bursty Arrivals, in Computer Networks and ISDN Systems, no. 24, 93-103.

Karol, M. J., Liu, Z. and Eng, K. (1995a) Distributed-queuing Request Update Multiple Access (DQRUMA) for Wireless Packet (ATM) Networks, in International Conference on Comm. Conference Record.

Karol, M. J., Liu, Z. and Eng, K. (1995b) An Efficient Demand-assignment Multiple Access Protocol for Wireless Packet (ATM) Networks, in Wireless Networks 1, 267-279.

LaMaire, R. , Krishna, A. and Ahmadi H. (1994) Analysis of a Wireless MAC Protocol with Client-Server Traffic and Capture, in IEEE J. Select. Areas in Comm., Vol.12, No 8, 1299-1313.

Li, M. and Chen, K. (1995) GRAPO - Optimized Group Randomly Addressed Polling for Wireless Data Networks, in Int. J. of Wireless Information Networks, Vol. 2, No. 4, 247-255.

Liu, Z., Karol, M. J., El Zarki, M. and Eng, K. Y. (1996) A Demand-Assignment Access Control for Multi-Code DS-CDMA Wireless Packet (ATM) Networks, in Proc. IEEE Infocom '96, 713-721.

Modiano, E. (1995) Dynamic Adaptive Multi-receiver Random Access Protocol for the Code Division Multiple Access Channel, Proc. of the 6th IEEE International Symp. on Personal, Indoor and Mobile Radio Comm. .

Narasimhan, P. and Yates, R. (1995) A New Protocol for the Integration of Voice and Data over PRMA, in Proc. of the 6th IEEE International Symp. on Personal, Indoor and Mobile Radio Comm., 623-627. 
Olivetti (1995) Olivetti Claims Radio ATM First, in Mobile Comm. International, Sept/Oct.

Papantoni-Kazakos, P., Likhanov, N. and Tsybakov, B. (1994)A Protocol for Random Multiple Access of Packets with Mixed priorities in Wireless Networks, in Proc. of the 5th IEEE International Symp. on Personal, Indoor and Mobile Radio Comm., 1121-1125.

Petras, D.(1995) Medium Access Control Protocol for transparent ATM Access in MBS, in RACE Mobile Telecomm. Summit.

Porter, J. and Hopper, A. (1994) An ATM based protocol for Wireless LANs, Olivetti Research's WWW-server <http://www.cam-orl.co.uk/index.html>, <ftp:// ftp.cam-orl.co.uk/pub/docs/ORL/tr.94.2.ps.Z>, links valid December 21997.

Porter, J., Hopper, A., Gilmurray, D., Mason, O., Naylon, J. and Jones, A. (1996) The ORL Radio ATM System, Architecture and Implementation, Olivetti Research's WWW-server <http://www.cam-orl.co.uk/index.html>, <ftp://ftp.cam-orl.co.uk/ pub/docs/ORL/tr.96.5.ps.Z>, links valid December 21997.

Raychaudhuri, D. (1996) Wireless ATM Networks: Architecture, System Design and Prototyping, in IEEE Personal Comm., Vol. 3, No 4, 42-49.

Roberts, L. (1975) ALOHA Packet System With and Without Slots and Capture, in Computer Comm. Review.

Smulders, P. and Blondia, C. (1994) Application of the Asynchronous Transfer Mode in Indoor Radio Networks, in Proc. of the 5th IEEE International Symp. on Personal, Indoor and Mobile Radio Comm., 839-843.

Stallings, W. (1994) Data and Computer Comm., 4th Edition, New York.

Sugihara, A., Enomoto, K. and Sasase, I. (1995) Throughput Performance of a Slotted Nonpersistent CSMA with an Adaptive Array, in Proc. of the 6th IEEE International Symp. on Personal, Indoor and Mobile Radio Comm., 633-637.

Tasaka, S. and Hayshi, K. (1995) Integrated Video and Data Transmission in the TDD ALOHA-reservation Wireless LAN, in International Conference on Comm. Conference Record.

Wen, J. and Wang, J. (1995) A New Protocol for Wireless Voice Comm. - Non-Collision Packet Reservation Multiple Access, in Proc. of the 6th IEEE International Symp. on Personal, Indoor and Mobile Radio Comm., 638-642.

Wilkinson, T., Phipps, T. and Barton, S. (1995) A Report on HIPERLAN Standardization", in Int. J. of Wireless Information Networks, Vol. 2, No. 2, 99-119.

Wu, G., Mukomoto, K., Fukuda, A., Mizuno, M. and Taira, K. (1995) A Dynamic TDMA Wireless Integrated Voice/Data System with Data Steal into Voice (DSV) Technique, in IEICE Trans. Com. Vol. E78-B, No 8, 1125-1135.

Zhuang, W. (1995) Medium Access Control Protocol for Multimedia Wireless Networks", in Proc. of the 6th IEEE International Symp. on Personal, Indoor and Mobile Radio Comm., 1094-1098. 